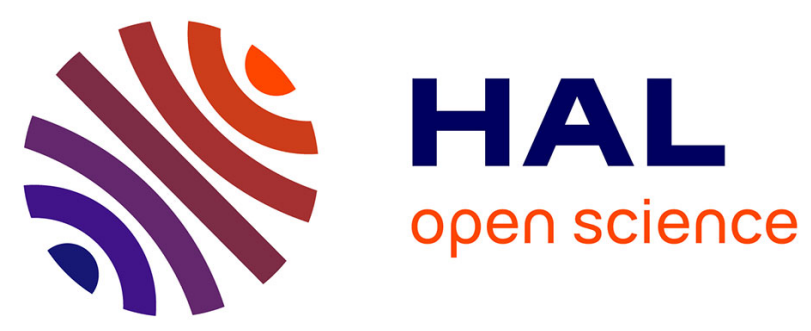

\title{
Quantitative relevance of substitutional impurities to carrier dynamics in diamond
}

Takaaki Shimomura, Yoshiki Kubo, Julien Barjon, Norio Tokuda, Ikuko Akimoto, Nobuko Naka

\section{- To cite this version:}

Takaaki Shimomura, Yoshiki Kubo, Julien Barjon, Norio Tokuda, Ikuko Akimoto, et al.. Quantitative relevance of substitutional impurities to carrier dynamics in diamond. Physical Review Materials, 2018, 2 (9), 10.1103/PhysRevMaterials.2.094601 . hal-02976733

\section{HAL Id: hal-02976733 \\ https://hal.science/hal-02976733}

Submitted on 26 Nov 2020

HAL is a multi-disciplinary open access archive for the deposit and dissemination of scientific research documents, whether they are published or not. The documents may come from teaching and research institutions in France or abroad, or from public or private research centers.
L'archive ouverte pluridisciplinaire HAL, est destinée au dépôt et à la diffusion de documents scientifiques de niveau recherche, publiés ou non, émanant des établissements d'enseignement et de recherche français ou étrangers, des laboratoires publics ou privés. 


\title{
Quantitative relevance of substitutional impurities to carrier dynamics in diamond
}

\author{
Takaaki Shimomura, ${ }^{1}$ Yoshiki Kubo, ${ }^{1}$ Julien Barjon, ${ }^{2}$ Norio Tokuda, ${ }^{3}$ Ikuko Akimoto, ${ }^{4}$ and Nobuko Naka ${ }^{1, *}$ \\ ${ }^{1}$ Department of Physics, Kyoto University, Kitshirakawa-Oiwake-cho, Sakyo-ku, Kyoto 606-8502, Japan \\ ${ }^{2}$ Groupe d'Etude de la Matière Condensée (GEMaC), Université de Versailles St-Quentin-en-Yvelines, CNRS, \\ Université Paris Saclay, 45 avenue des Etats-Unis, 78035 Versailles Cedex, France \\ ${ }^{3}$ Nanomaterials Research Institute, Kanazawa University, Kakuma-machi, Kanazawa 920-1192, Japan \\ ${ }^{4}$ Department of Materials Science and Chemistry, Wakayama University, Wakayama 640-8510, Japan
}

(Received 25 May 2018; published 4 September 2018)

\begin{abstract}
We have quantified substitutional impurity concentrations in synthetic diamond crystals down to sub-partsper-billion levels. The capture lifetimes of electrons and excitons injected via photoexcitation were compared for several samples with different impurity concentrations. Based on the assessed impurity concentrations, we have determined the capture cross section of electrons for boron impurity, $\sigma_{\mathrm{A}}=1 \times 10^{-14} \mathrm{~cm}^{2}$, and that of excitons for nitrogen impurity, $\sigma_{\mathrm{D}}^{\text {ex }}=3 \times 10^{-14} \mathrm{~cm}^{2}$. The general tendency of the mobility values for different carrier species is successfully reproduced by including carrier scattering by impurities and excitons.
\end{abstract}

DOI: 10.1103/PhysRevMaterials.2.094601

\section{INTRODUCTION}

Diamond is a wide-gap material attractive for various applications because of its unique physical and chemical properties. Its practical applications range from ultraviolet light-emitting diodes, radiation detectors, and single-photon sources, to biosensors. Owing to the recent progress of crystal growth techniques, such as the chemical-vapor-deposition (CVD) and high-pressure high-temperature (HPHT) methods, high-purity single crystals of diamond are becoming commercially available [1]. It has been known that the dominant substitutional impurities in synthetic diamond are nitrogen and boron owing to the proximity of their atomic sizes to that of carbon. The nitrogen impurities in diamond affect not only the spin relaxation time of electrons localized at nitrogen-vacancy centers [2], but also the relaxation dynamics of free excitons [3].

To quantify nitrogen or boron impurities in diamond at parts-per-million (ppm) levels, one can utilize absorption [4] and secondary-ion mass spectroscopy (SIMS). However, methods for evaluating nitrogen at parts-per-billion (ppb) levels below the SIMS detection limits are not common [2]. The quantification method for boron impurities using cathodoluminescence [5] has been recently extended with a detection limit below $0.05 \mathrm{ppb}[6,7]$. Owing to the long-term difficulty of doping control and the quantification problem, capture cross sections of carriers or excitons for impurities in diamond have been missing parameters for 20 years [8,9].

In this study, we have performed electron-paramagneticresonance (EPR) measurements to determine the concentrations of substitutional nitrogen down to sub-ppb levels. Furthermore, boron impurities with concentrations below $2 \mathrm{ppb}$ were evaluated using careful photoluminescence analysis. Based on the assessed impurity concentrations, we quantitatively discuss the momentum relaxation times of charge

*naka@scphys.kyoto-u.ac.jp carriers, which determine the carrier mobility, in addition to the capture cross sections of charge carriers and excitons. The extraction of the cross sections was enabled by our careful quantification of impurity concentrations. The capture lifetimes were observed to be limited by the interactions with impurities in high-purity diamond. The obtained parameter values are critical both for modeling of electronic devices and for defect engineering using color centers, which is an emerging field in solid-state physics.

\section{EXPERIMENTS}

Five samples with unknown impurity concentrations were used in the experiments, along with two standard samples (see Table I for nitrogen and Table II for boron quantification). The first letter of the sample names $(\mathrm{C}$ or $\mathrm{H})$ designates the growth method, i.e., CVD or HPHT. C1 (C6) is an optical (electronic)grade single-crystal diamond purchased from ElementSix, Ltd. $\mathrm{H} 7$ is a dislocation-free and single-sector substrate purchased from Technical Institute for Superhard and Novel Carbon Materials. The standard sample (H2) for nitrogen quantification was cross evaluated using infrared absorption and SIMS, and the concentration of charge-neutral substitutional nitrogen was $\left[\mathrm{N}_{\mathrm{s}}^{0}\right]=23 \pm 2 \mathrm{ppm}\left(=4.1 \times 10^{18} \mathrm{~cm}^{-3}\right)$. The inhomogeneity of the dopant concentration was particularly considered, i.e., we obtained an average value of the infrared absorption measured in five different portions within the sample under a microscopic Fourier-transform infrared spectrometer (Bruker, VERTEX80V). The other standard sample $(\mathrm{C} 0)$ for boron quantification is an epitaxial layer of thickness $13 \mu \mathrm{m}$ grown on a type $\mathrm{Ib}$ diamond substrate. The boron concentration in the epitaxial layer was characterized using electrical measurements and SIMS along the depth [10]. The boron concentration averaged over the layer thickness was $\left[\mathrm{B}_{\mathrm{s}}\right]=102 \mathrm{ppb}(=$ $\left.1.8 \times 10^{16} \mathrm{~cm}^{-3}\right)$. For the samples examined (C6-H5), the highest possible concentrations quoted by the suppliers were $50 \mathrm{ppb}$ for boron and $100 \mathrm{ppb}$ for nitrogen [11]. 
TABLE I. List of the samples used in the experiments. The EPR signal intensity $I_{\mathrm{EPR}}$, sample weight $W$, cavity quality factor $Q$, $I_{\mathrm{EPR}}$ divided by the square root of the microwave power $P$, and concentration $\left[\mathrm{N}_{\mathrm{s}}^{0}\right]$ of charge-neutral substitutional nitrogen are given.

\begin{tabular}{lccccc}
\hline \hline Sample & $I_{\mathrm{EPR}}$ & $W(\mathrm{mg})$ & $Q$ & $I_{\mathrm{EPR}} / \sqrt{P}$ & {$\left[\mathrm{~N}_{\mathrm{s}}^{0}\right](\mathrm{ppb})$} \\
\hline C6 & 0.0277 & 32.1 & 4700 & 0.0013 & $0.07 \pm 0.02$ \\
H7 & 0.121 & 4.22 & 6100 & 0.0079 & $2.7 \pm 0.5$ \\
C1 & 1.37 & 10.5 & 5100 & 0.083 & $14 \pm 1$ \\
H6 & 18.2 & 15.6 & 5500 & 0.89 & $94 \pm 7$ \\
H5 & 73.4 & 18.0 & 7600 & 2.2 & $240 \pm 20$ \\
H2 & 343 & 3.88 & 6800 & 66 & $(23 \pm 2) \times 10^{3}$ \\
\hline \hline
\end{tabular}

A substitutional nitrogen atom in diamond forms a nitrogen $\mathrm{P}_{1}$ center. Two spin levels of $s=1 / 2$ arise according to the Zeeman effect under an applied magnetic field. Each level is further split into triplets by the hyperfine interaction with a nuclear spin $(I=1)$ originating from ${ }^{14} \mathrm{~N}$ atoms of a natural abundance of $99.64 \%$. The concentration of unpaired electrons, or that of the $\mathrm{P}_{1}$ centers, can be determined based on the microwave absorption associated with the transitions between these electronic levels [12]. The EPR signal was measured with X-band microwave at a frequency of $9.6 \mathrm{GHz}$ for samples mounted in a dielectric cavity (Bruker, MD-5-W1, TE011) at $295 \mathrm{~K}$. The measurement was performed in the continuous-wave regime by using the spectrometer (Bruker, ELEXSYS E580). The nitrogen concentration was obtained by using the following relation:

$$
\left[N_{\mathrm{s}}^{0}\right] \propto I_{\mathrm{EPR}} /(W Q \sqrt{P}),
$$

where $I_{\mathrm{EPR}}, W, Q$, and $P$ represent the EPR signal intensity, weight of the sample, cavity quality factor, and microwave power, respectively. The EPR signal intensity was evaluated at the limit of weak microwave power to avoid the saturation effect. This was facilitated by analyzing the power dependence of the EPR signals and subsequently choosing appropriate microwave power for each sample for quantification (see Table I). The absolute concentration was evaluated by using the signal intensity from the standard sample $(\mathrm{H} 2)$.

TABLE II. Photoluminescence intensity ratio, $R$, of free and boron-bound excitons at the effective exciton temperature of $12 \mathrm{~K}$ and the boron concentration $\left[\mathrm{B}_{\mathrm{s}}\right] . N_{\mathrm{ii}}=2 \min \left(\left[\mathrm{B}_{\mathrm{s}}\right],\left[\mathrm{N}_{\mathrm{s}}^{0}\right]\right)$ is the expected concentration of ionized impurities owing to compensation at thermodynamic equilibrium. $\mathrm{H} 7$ is a single-sector crystal, whereas $\mathrm{H} 6$ and $\mathrm{H} 5$ consist of multiple sectors. (*The value is for the position of PL decay measurement.)

\begin{tabular}{lccr}
\hline \hline Sample & $R$ & {$\left[\mathrm{~B}_{\mathrm{s}}\right](\mathrm{ppb})$} & \multicolumn{1}{c}{$N_{\mathrm{ii}}\left(\mathrm{cm}^{-3}\right)$} \\
\hline C6 & $<0.0005$ & $<0.13$ & $<2.5 \times 10^{13}$ \\
H7 & 0.0062 & 1.5 & $5.3 \times 10^{14}$ \\
C1 & $<0.0015$ & $<0.4$ & $<1.4 \times 10^{14}$ \\
H6 & 0.027 & 7.2 & $2.5 \times 10^{15}$ \\
H5 & $0.031 \pm 0.003$ & $8.4 \pm 1$ & $3.0 \times 10^{15}$ \\
C0 & 0.38 & 102 & \\
\hline
\end{tabular}

The boron concentration $\left[\mathrm{B}_{\mathrm{s}}\right]$ was determined based on the photoluminescence (PL) intensity of boron-bound excitons relative to that of free excitons [6]. The excitation source was light of wavelength $213 \mathrm{~nm}$ from a tunable laser based on an optical-parametric-oscillator system (Ekspla, NT242). The pulse duration was $2.5 \mathrm{~ns}$ and the repetition rate was $1 \mathrm{kHz}$. Instead of the continuous excitation used in Ref. [6], pulsed excitation was used here but with a minimized laser power (e.g., $0.4 \mu \mathrm{W}$ ), in order to achieve suitable conditions for the quantification. The measurements were performed at $7 \mathrm{~K}$ for samples mounted in a closed-cycle cryostat. The PL signal was detected using a charge-coupled device camera (Andor, DU940N-BU2) at the exit of a monochromator (Horiba Jobin Yvon, iHR-550, 2400 grooves $/ \mathrm{mm}$ grating). The entrance slit of the monochromator was set at $150 \mu \mathrm{m}$ to achieve sufficient signal intensities under weak excitation. The absolute boron concentration was determined by comparing the PL intensity ratio with that of the standard sample $(\mathrm{C} 0)$ measured under identical conditions.

\section{RESULTS AND DISCUSSION}

\section{A. Impurity quantification}

Figure 1 shows the EPR spectra obtained from each sample. Here, we focus on the $(-1 / 2,0) \rightarrow(1 / 2,0)$ transition, where the numbers in parentheses indicate the electron spin and nuclear spin. The center magnetic field $B_{0}$ corresponds to $g=2.0027 \pm 0.0002$. The spectral shape of the EPR signals is generally given by a Lorentzian function, but here it is represented in a differential form owing to the modulation of the magnetic field (modulation width $=0.1 \mathrm{G}$ ) to improve
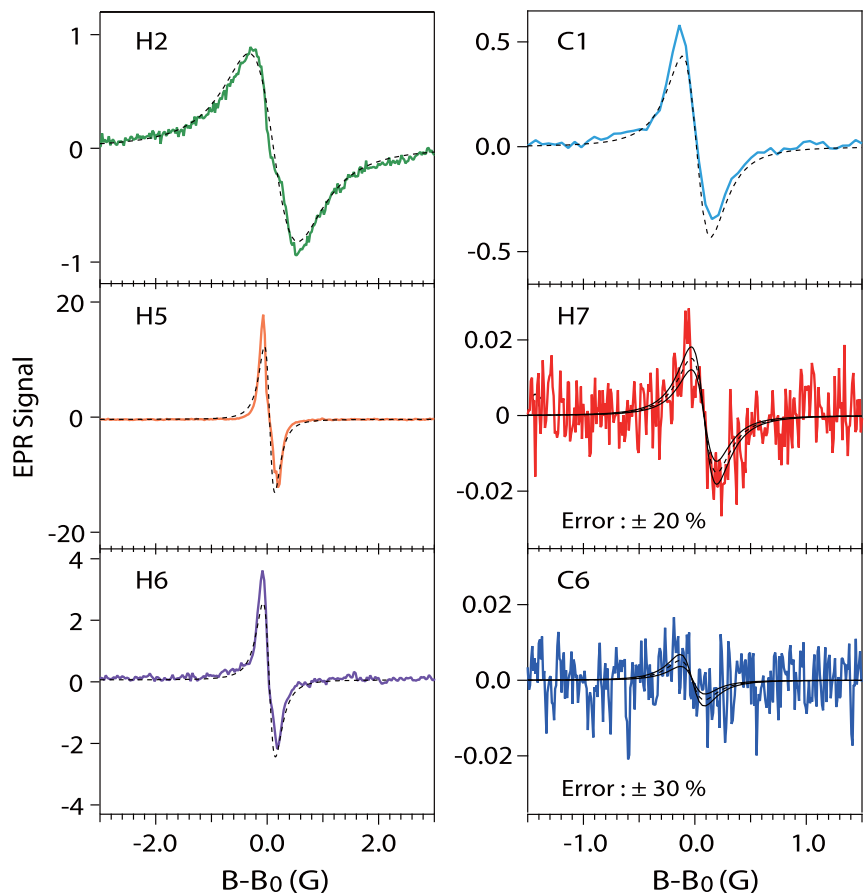

FIG. 1. EPR spectra of the $(-1 / 2,0) \rightarrow(1 / 2,0)$ transition of the substitutional nitrogen $\left(\mathrm{P}_{1}\right)$ center in various samples. Dashed lines are the best-fit functions. Thin full lines are drawn to estimate the errors $(\epsilon= \pm 0.2$ for $\mathrm{H} 7$ and $\epsilon= \pm 0.3$ for $\mathrm{C} 6)$. 
the sensitivity. The EPR signal intensity $I_{\mathrm{EPR}}$ in Eq. (1) is obtained by integrating a raw signal twice over the magnetic field strength. Alternatively, $I_{\mathrm{EPR}}=\pi a b$ is obtained by fitting the data with a differentiated spectral function

$$
L^{\prime}(B)=-a b^{2} \frac{B-B_{0}}{\left[b^{2}+\left(B-B_{0}\right)^{2}\right]^{2}} .
$$

The dashed lines represent the best-fit functions. The evaluated signal intensity $I_{\mathrm{EPR}}$ and estimated nitrogen concentration $\left[\mathrm{N}_{\mathrm{s}}^{0}\right]$ are summarized in Table I. The errors for a concentration below the ppb level were carefully evaluated by comparing the data with the best-fit function magnified by a factor of $(1+\epsilon)$, where $\epsilon$ represents the relative uncertainty. These spectral functions are shown by thin full lines in Fig. 1. The minimum nitrogen concentration estimated was of the order of $0.1 \mathrm{ppb}$, which corresponds to $1.76 \times 10^{13} \mathrm{~cm}^{-3}$.

Regarding the boron concentration, we referred to the previous publication [6] on the ratio $R$ of cathodoluminescence from bound and free excitons in boron-doped diamond. To reduce errors during the quantification procedure, we mounted three samples in the same holder for comparison. The obtained PL intensity ratio and boron concentration are summarized in Table II. The PL from bound excitons was below the detection limit in $\mathrm{C} 1$ and $\mathrm{C} 6$; thus we presented an upper bound for the boron concentration in Table II by considering the signal-to-noise ratio.

\section{B. Capture lifetimes}

The above result indicates that the nitrogen concentration is higher than the boron concentration in most samples. By using these assessed samples having different impurity concentrations, we discuss the influence of impurities on the capture lifetimes of excitons or carriers. Figure 2(a) shows the normalized intensity of cyclotron resonance signals for electrons at $10 \mathrm{~K}$ in various samples measured in our previous work under photoexcitation of approximately $223 \mathrm{~nm}[13,14]$. The decay time was extracted as $1 / e$ time of an exponential decay function convolved with a Gaussian responsible for the excitation pulse width (5 ns) and the system response function. The decay time largely depended on the samples. Since the radiative lifetime of carriers in diamond is much longer, the decay time is determined by the nonradiative rate, which is considered to be dominated by capture for impurities. Figure 2(b) shows a plot of the measured decay time as a function of the total impurity concentration, i.e., the horizontal axis of the figure is $\left[N_{\mathrm{s}}^{0}\right]+\left[\mathrm{B}_{\mathrm{s}}\right]$. The error bars include quantification errors (see Tables I and II) and possible sector-dependent impurity concentrations for H6. The capture lifetimes are calculated as

$$
t=\frac{1}{v\left(\sigma_{\mathrm{A}} n_{\mathrm{A}}+\sigma_{\mathrm{D}} n_{\mathrm{D}}\right)},
$$

where $v=\sqrt{3 k_{B} T / m_{\mathrm{dos}}^{*}}\left(m_{\mathrm{dos}}^{*}=0.496 m_{0}\right.$ [15]) is the electron thermal velocity determined by the temperature $T, \sigma_{\mathrm{A}}$ and $\sigma_{\mathrm{D}}$ are the cross sections of electrons against capture for boron and nitrogen, respectively, and $n_{\mathrm{A}}$ and $n_{\mathrm{D}}$ are the acceptor or donor concentrations, respectively. We consider $n_{\mathrm{A}}=\left[B_{\mathrm{S}}\right]$ and $n_{\mathrm{D}}=$ $\left[N_{\mathrm{s}}^{0}\right]$ by assuming neutralization of compensated impurities by photoexcited carriers as proposed in Refs. [16,17].
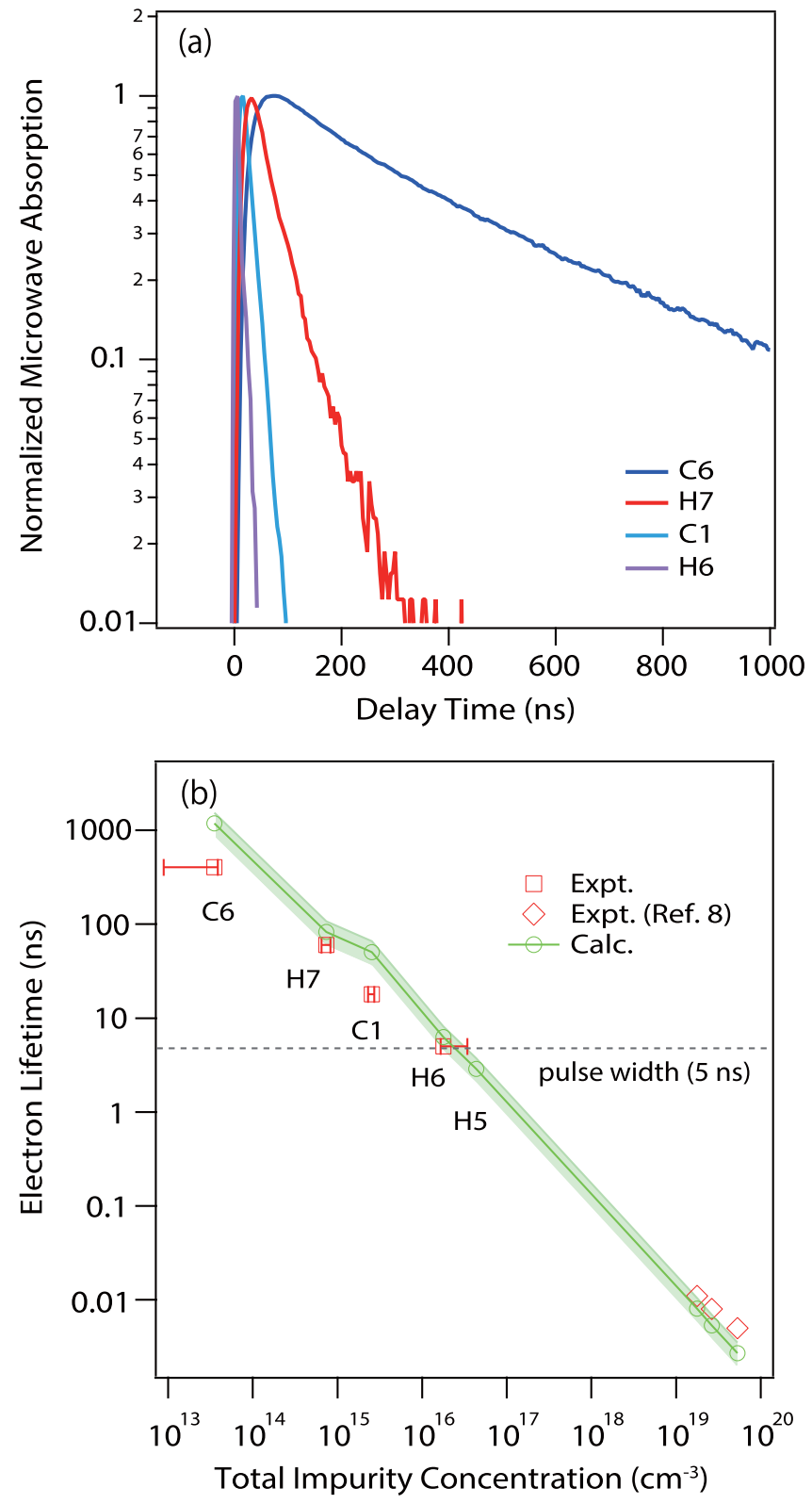

FIG. 2. (a) Normalized intensity of the cyclotron resonance signal for electrons at $10 \mathrm{~K}$ in various samples. (b) Plot of electron lifetime as a function of total impurity concentration. The lines connect the calculated points $\left(\sigma_{\mathrm{A}}=1.1 \times 10^{-14} \mathrm{~cm}^{2}\right.$ and $\sigma_{\mathrm{D}}=2.3 \times 10^{-15} \mathrm{~cm}^{2}$ with the shaded area representing $\pm 30 \%$ errors) as a guide for the eyes.

According to the model of classical collision, a cross section is given by $\sigma_{\mathrm{A}(\mathrm{D})}=\pi a_{\mathrm{A}(\mathrm{D})}^{2}$. The effective Bohr radii of the impurity states are given by $a_{\mathrm{A}}=\hbar / \sqrt{2 m_{\mathrm{oh}} E_{\mathrm{A}}}=5.2 \AA$ and $a_{\mathrm{D}}=\hbar / \sqrt{2 m_{\mathrm{oe}} E_{\mathrm{D}}}=2.4 \AA$, where $E_{\mathrm{A}(\mathrm{D})}=0.37(1.7) \mathrm{eV}$ is the acceptor (donor) activation energy and $m_{\mathrm{oh}}\left(m_{\mathrm{oe}}\right)=$ $0.377(0.385) m_{0}$ is the conductivity effective mass for holes (electrons). Here, a hydrogen model was assumed both for boron and nitrogen levels. Considering the scaling relation $\sigma_{\mathrm{A}}=\sigma_{\mathrm{D}} a_{\mathrm{A}}^{2} / a_{\mathrm{D}}^{2}, \sigma_{\mathrm{A}}=(1.1 \pm 0.3) \times 10^{-14} \mathrm{~cm}^{2}$ and $\sigma_{\mathrm{D}}=$ $(2.3 \pm 0.7) \times 10^{-15} \mathrm{~cm}^{2}$ are obtained for good consistency between the calculated capture times (circles) and data (squares). 
The latter cross section has reasonable consistency with the value measured using terahertz time-domain spectroscopy [8] on electron capture for nitrogen, $1.4 \times 10^{-15} \mathrm{~cm}^{2}$ (or $1.7 \times 10^{-15} \mathrm{~cm}^{2}$ after correction of the difference in the assumed effective mass values). The measured lifetime for C6 was shorter than the calculated one assuming the boron concentration at the upper bound. This indicates the existence of other kinds of structural defects or impurities, such as hydrogen, silicon, interstitials, or dislocations, acting as a center capturing electrons in the CVD-grown diamond. Further, because of the larger cross section for boron than for nitrogen, the electron capture time more sensitively depends on the boron concentration. Hence the plot does not appear linear against the total impurity concentration.

Figure 3(a) shows the normalized PL intensity of freeexciton luminescence following the pulsed excitation for $2.5 \mathrm{~ns}$ in $\mathrm{C} 1, \mathrm{H} 7$, and $\mathrm{C} 6$ at $7 \mathrm{~K}$. The detailed experimental setup for this measurement is described elsewhere [18]. After the fast decay owing to many-body effects, the $1 / e$ time of the second decay component indicates the time required for free excitons to be captured by impurities. The capture lifetimes were obtained as 4.8, 28, and $119 \mathrm{~ns}$ for $\mathrm{C} 1, \mathrm{H} 7$, and C6, respectively. The analysis for decay times shorter than the duration of the laser pulse involves large errors because separating many-body effects is not straightforward. Nevertheless, there is an apparent tendency that the capture lifetime was shorter for a sample with a higher concentration of impurities. Figure 3(b) shows a plot of the capture lifetime as a function of the total impurity concentration. The calculations were performed based on Eq. (3), where $v=\sqrt{3 k_{B} T / m_{\mathrm{ex}}^{*}}$ is the exciton thermal velocity with the translational effective mass $m_{\mathrm{ex}}^{*}=0.76 m_{0}[15]$ and $\sigma_{\mathrm{A}}$ and $\sigma_{\mathrm{D}}$ should be replaced by the cross sections of excitons, $\sigma_{\mathrm{A}}^{\mathrm{ex}}$ and $\sigma_{\mathrm{D}}^{\mathrm{ex}}$, respectively. We assumed $\sigma_{\mathrm{A}}^{\mathrm{ex}}=3.4 \times 10^{-14} \mathrm{~cm}^{2}$ according to Ref. [9] for boron, where a factor of $\sqrt{8 /(3 \pi)}$ was multiplied to correct the difference in the definitions of thermal velocity. The cross section for nitrogen is not exactly known, because the direct measurement is difficult owing to the fast Auger process. For the calculation shown by circles, we used $\sigma_{\mathrm{D}}^{\text {ex }}=3.4 \times 10^{-14} \mathrm{~cm}^{2}$ as the capture cross section for nitrogen and obtained a reasonable consistency with the data.

Here, we would like to compare the obtained cross sections for carriers and excitons. The electron cross section for boron is larger than that for nitrogen and both are smaller than the exciton cross sections. This indicates that electrons have fewer chances to be captured by impurities than excitons. The difference appears to originate from the difference in the Bohr radius $\left(a_{\mathrm{A}}, a_{\mathrm{D}}\right)$ of carriers and that $\left(a_{\mathrm{B}}^{\mathrm{ex}}\right)$ of excitons. By assuming classical collision between hard spheres, the Bohr radius scales proportionally to $\sqrt{\sigma}$. Therefore, the excitonic Bohr radius is roughly estimated as $a_{\mathrm{B}}^{\mathrm{ex}}=a_{\mathrm{A}} \sqrt{\sigma_{\mathrm{A}}^{\mathrm{ex}} / \sigma_{\mathrm{A}}}$ by using the acceptor cross sections and $a_{\mathrm{B}}^{\mathrm{ex}}=a_{\mathrm{D}} \sqrt{\sigma_{\mathrm{D}}^{\mathrm{ex}} / \sigma_{\mathrm{D}}}$ by using the donor cross sections. Both relations coherently lead to an exciton Bohr radius of $9.2 \AA$. Considering the crudeness of the hydrogen model, this value is not far from the value, $a_{\mathrm{B}}^{\mathrm{ex}}=$ $13.7 \AA$, derived from the exciton binding energy of $92 \mathrm{meV}$ [19]. Therefore, exciton capture is determined by the exciton Bohr radius rather than by the effective radius of impurities.

The good consistency obtained above between the experimental data and theory indicates that neglecting ionized
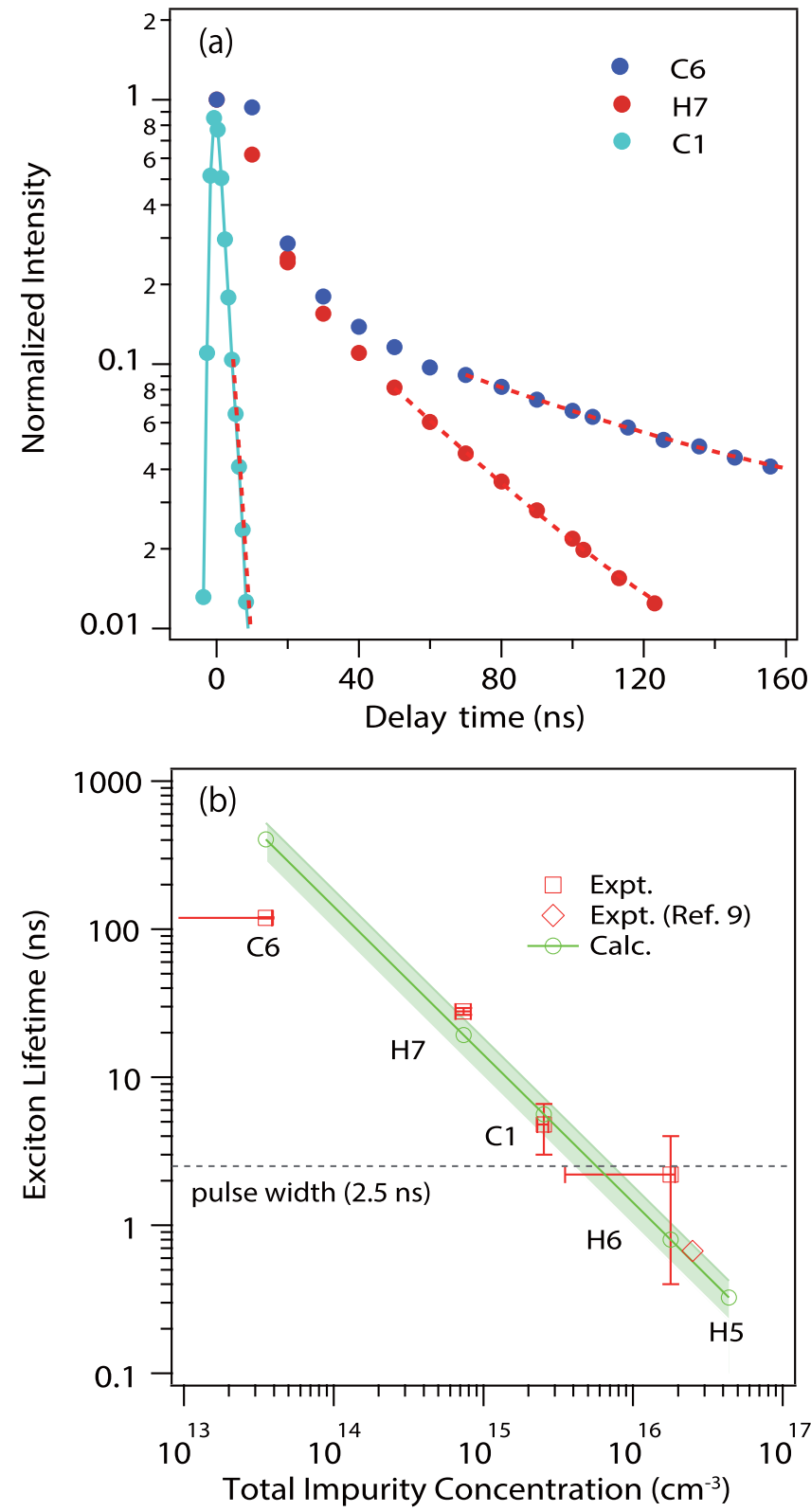

FIG. 3. (a) Normalized PL intensity of free-exciton luminescence in $\mathrm{C} 1, \mathrm{H} 7$, and $\mathrm{C} 6$ at $7 \mathrm{~K}$. (b) Plot of exciton lifetime as a function of the total impurity concentration. The line connects the calculated points $\left(\sigma_{\mathrm{A}}^{\mathrm{ex}}=\sigma_{\mathrm{D}}^{\mathrm{ex}}=3.4 \times 10^{-14} \mathrm{~cm}^{2}\right.$ with the shaded area representing $\pm 30 \%$ errors) as a guide for the eyes.

impurities is reasonable. Additionally, capture by impurity complexes such as nitrogen-vacancy centers should not be significant because the density of the centers is small, i.e., at least $1 / 200$ times $\left[\mathrm{N}_{\mathrm{s}}^{0}\right][20]$, and the cross section is known to be very small $\left(10^{-15} \mathrm{~cm}^{2}\right)$ [21]. The excitons in diamond are bound by neutral impurities but unbound by charged impurities owing to the relatively small mass imbalance between electrons and holes [22].

\section{Momentum relaxation time of charge carriers}

In this subsection, we compare the momentum relaxation time (or the scattering time, in short) of charge carriers 


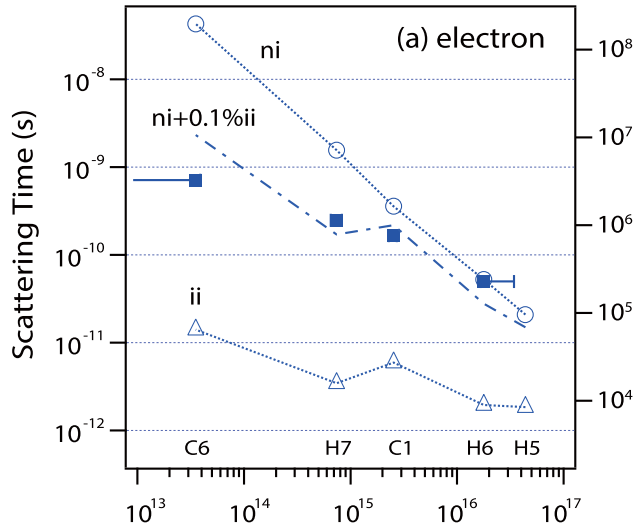

Total Impurity Concentration $\left(\mathrm{cm}^{-3}\right)$

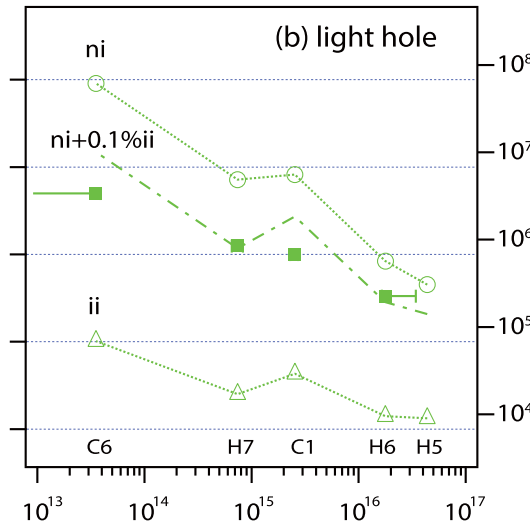

Total Impurity Concentration $\left(\mathrm{cm}^{-3}\right)$

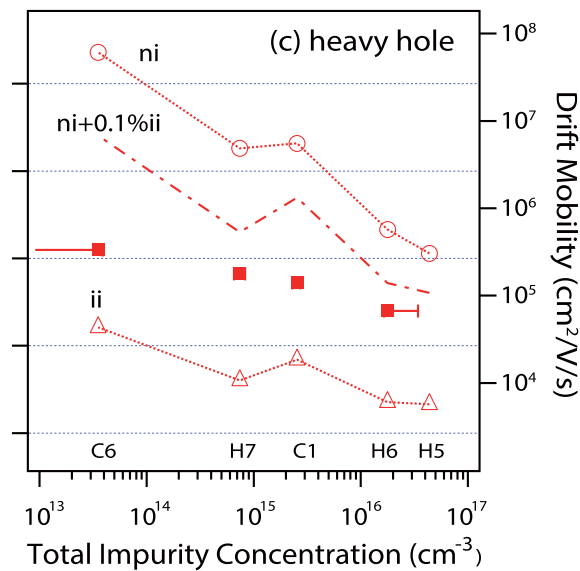

Total Impurity Concentration $\left(\mathrm{cm}^{-3}\right)$
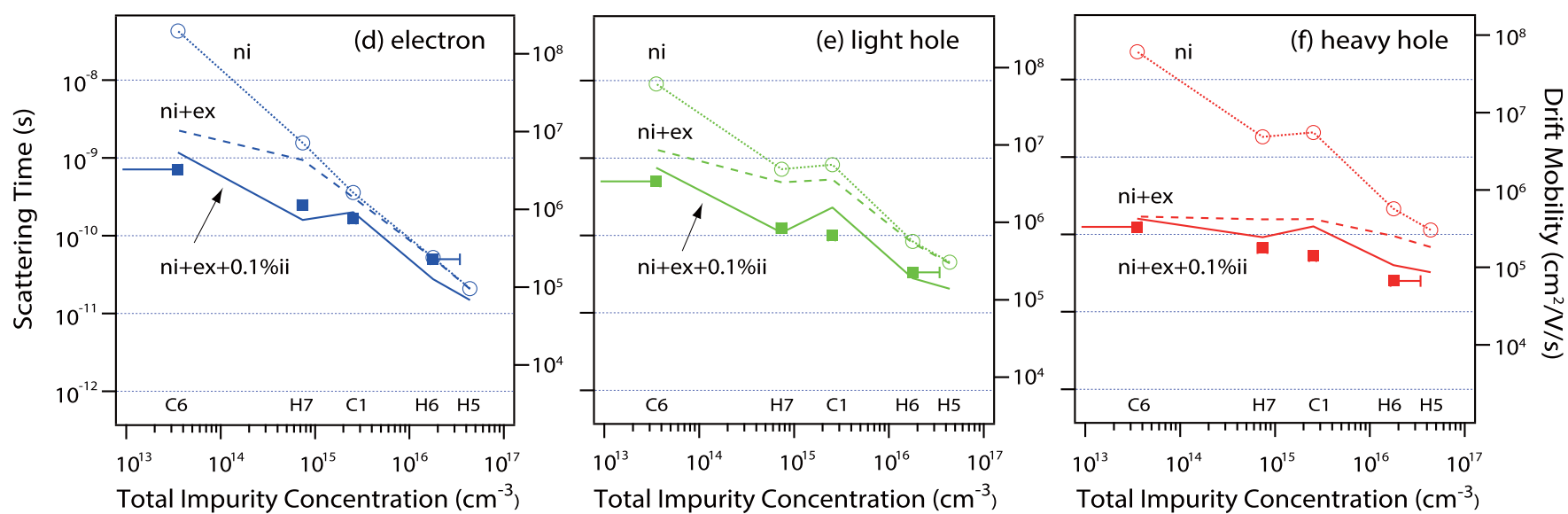

FIG. 4. Solid squares: scattering times measured at $10 \mathrm{~K}$ in different samples for (a),(d) electron, (b),(e) light hole, and (c),(f) heavy hole. Left axes and grids are common for all six panels. Symbols connected by lines represent the calculations of neutral impurity (ni) and ionized impurity (ii) scattering. The dashed dotted lines in (a),(b),(c) represent the sum of (ni) and (ii) scattering calculated with $0.1 \% N_{\mathrm{ii}}$. The dashed lines in (d),(e),(f) are the sum of (ni) and exciton (ex) scattering and the full lines are the sum of (ni), (ex), and (ii) scattering with $0.1 \% N_{\mathrm{ii}}$.

in different samples. The thermal average of the scattering time, $\tau$, is directly related to drift mobility by the relation $\mu=e \tau / m_{\mathrm{c}}^{*}$, where $e$ is the elementary charge and $m_{\mathrm{c}}^{*}$ is the conductivity effective mass. According to Mathiessen's rule, the scattering time is approximated by the inverse of the sum of the rates owing to intrinsic and extrinsic scattering processes. The intrinsic process is well known to be dominated by phonon scattering [23]. If the extrinsic process is dominated by impurity scattering, one can, in principle, predict the drift mobility of charge carriers in assessed samples, after precisely knowing the impurity concentrations.

The scattering time was measured by using cyclotron resonance [13], where the ratio of the resonance width to the resonance magnetic field yields the value of $\tau$. With a decrease in temperature $T$, the scattering time $\tau$ increased by $T^{-3 / 2}$ down to $50 \mathrm{~K}$, consistent with the acoustic-phonon scattering theory. At lower temperatures, $\tau$ approached a constant value with a weaker temperature dependence than $T^{-3 / 2}$. The low-temperature scattering time was shorter for diamond containing impurities of higher concentrations (see the plot of $1 / \tau$ in Fig. 12 of Ref. [13]).

The scattering times at $10 \mathrm{~K}$ in the assessed samples are plotted using solid squares in Figs. 4(a)-4(c), respectively, for the electron, light hole, and heavy hole. The longest scattering time was measured for the electron in C6, whereas the shortest value was measured for the heavy hole in H6. The resonance spectra in H5 were too broad to extract accurate scattering time. The right axis of each panel represents the mobility value obtained by using the conductivity effective masses, $m_{\mathrm{oe}}^{*}=$ $0.385 m_{0}, m_{\mathrm{lh}}^{*}=0.26 m_{0}$, and $m_{\mathrm{hh}}^{*}=0.67 m_{0}$, respectively, for the electron, light hole, and heavy hole.

The most apparent contribution to charge carrier scattering is from neutral impurities (ni), whose theoretical scattering time is given by the Erginsoy formula [24] for an electronhydrogen scattering model and its modification to a positronhydrogen scattering picture by Otsuka, Murase, and Iseki [25]. Thus

$$
\tau_{\text {ni-e }}=m_{\text {dos }}^{*} /\left(3.4 n_{\mathrm{A}} a_{\mathrm{A}} \hbar+20 n_{\mathrm{D}} a_{\mathrm{D}} \hbar\right) \propto m_{\mathrm{dos}}^{*},
$$

for an electron, and

$$
\tau_{\mathrm{ni}-\mathrm{h}}=m_{\mathrm{hh}(\mathrm{hh})}^{*} /\left(20 n_{\mathrm{A}} a_{\mathrm{A}} \hbar+3.4 n_{\mathrm{D}} a_{\mathrm{D}} \hbar\right) \propto m_{\mathrm{hh}(\mathrm{hh})}^{*},
$$

for a hole. As shown by the circles in Figs. 4(a)-4(c), the calculated scattering times for (ni) are longer than the experimental data, especially for the heavy hole. In the following paragraphs, we discuss the other possible contributions.

The short scattering times measured for the heavy hole could be due to the fluctuation in the isotopic composition 
of natural carbon $\left(98.9 \%{ }^{12} \mathrm{C}\right.$ and $\left.1.1 \%{ }^{13} \mathrm{C}\right)$. The isotope scattering is modeled using alloy scattering [26] with the scattering time expressed as

$$
\tau_{\text {iso }}=\frac{\left(2 \pi \hbar^{2}\right)^{2}}{\pi m_{\mathrm{dos}}^{* 2}}\left(\frac{d E_{c}}{d x}\right)^{-2} \frac{1}{a_{0}^{3} x \sqrt{3 k_{B} T / m_{\mathrm{dos}}^{*}}} \propto m_{\mathrm{dos}}^{*-3 / 2},
$$

where $x=0.011$ is the fraction of ${ }^{13} \mathrm{C}, a_{0}=3.57 \AA$ is the lattice constant of diamond, and $d E_{c} / d x=14.6 \mathrm{meV}$ is the isotopic gap shift [27]. The calculated scattering times are $\tau_{\text {iso }}=1.5 \times 10^{-7} \mathrm{~s}, 2.2 \times 10^{-7} \mathrm{~s}$, and $5.5 \times 10^{-8} \mathrm{~s}$ for the electron, light hole, and heavy hole, respectively. As these values are much larger than the experimental data, we conclude that their contributions to the mobility are not significant.

A local strain could also be a source of carrier scattering. An order-of-magnitude scattering time is in the range of $10^{-10}$ s according to Eq. (3) of Ref. [28]:

$$
\tau_{\text {strain }}=\frac{32}{3 \pi}\left(\frac{1-v}{1-2 v}\right)^{2} \frac{k_{B} \hbar}{\Xi^{2} \lambda^{2} N} T,
$$

where $v=0.18$ is Poisson ratio, $\Xi=8.7(10) \mathrm{eV}$ is the deformation potential for the electron (light and heavy holes) [23], and $\lambda$ is the unit crystallographic slip distance considered to be equal to the lattice constant. Here, we assumed dilations around edge-type dislocations at a density of $N=10^{5} \mathrm{~cm}^{-2}$, which is a typical value in samples with the same grade as C6 and orders of magnitude greater than that in HPHT samples (H7 is dislocation free). The theoretical scattering time is independent of the effective mass and does not explain the discrepancy between (ni) and squares in Fig. 4.

The case of ionized impurities (ii) deserves to be considered as the samples are compensated. The calculated scattering times for the ionized impurity interaction according to the Conwell-Weisskopf formula [29],

$$
\tau_{\mathrm{ii}}=\frac{64 \sqrt{\pi m_{\mathrm{dos}}^{*}} \epsilon^{2}\left(2 k_{B} T\right)^{3 / 2}}{e^{4} N_{\mathrm{ii}} \ln \left[\left(1+144 \pi^{2} \epsilon^{2} k_{B}^{2} T^{2} /\left(e^{4} N_{\mathrm{ii}}^{2 / 3}\right)\right]\right.} \propto m_{\mathrm{dos}}^{* 1 / 2},
$$

are represented by triangles in Figs. 4(a)-4(c) when using the density $N_{\mathrm{ii}}=2 \min \left(\left[\mathrm{B}_{\mathrm{s}}\right],\left[\mathrm{N}_{\mathrm{s}}^{0}\right]\right)$ as listed in Table II [30] and the dielectric constant $\epsilon=5$.7. With such inputs, the calculated (ii) scattering times are approximately two orders of magnitude shorter than the measured ones. This result indicates that the actual density of ionized impurities is much smaller than $N_{\text {ii }}$. The dashed dotted lines in Figs. 4(a)-4(c) are instead calculated with the neutral impurities and the concentration of ionized impurities equal to $0.1 \% N_{\text {ii }}$. The relatively good consistency with the experimental data indicates that at least $99.9 \%$ of the compensated impurities are neutralized by photoexcited carriers, consistent with the assumption made in subsection $\mathrm{B}$ for the analysis of capture lifetimes. Our result also broadens the context known from the first experiments in silicon [16], where ionized impurity scattering plays a minor role in cyclotron resonance under photoexcitation compared with Hall-effect measurements.

Finally, we had to consider the scattering between free carriers and excitons [31] because the short scattering times for the heavy hole are still not correctly described with both neutral and ionized impurities. The carrier-exciton collision occurs with a scattering time $\tau_{\mathrm{ex}}=1 /\left(Q n_{\mathrm{ex}} v\right)$, where $Q$ is the exciton-carrier cross section. The cross section depends on the mass ratio of scattering particles and thus on the carrier species. Our estimates based on the literature [32] yield $Q_{\mathrm{e}}=2.6 \pi\left(a_{\mathrm{B}}^{\mathrm{ex}}\right)^{2}=1.4 \times 10^{-13} \mathrm{~cm}^{2}, Q_{\mathrm{lh}}=$ $3 \pi\left(a_{\mathrm{B}}^{\mathrm{ex}}\right)^{2}=1.6 \times 10^{-13} \mathrm{~cm}^{2}$, and $Q_{\mathrm{hh}}=42 \pi\left(a_{\mathrm{B}}^{\mathrm{ex}}\right)^{2}=2.2 \times$ $10^{-12} \mathrm{~cm}^{2}$. The exciton-carrier cross section for the heavy hole is observed to be one order of magnitude higher than that for the electron or light hole. This can be understood by the fact that the interaction potential for exchange of the incident carrier and that of the exciton becomes repulsive when the mass ratio exceeds unity, which is the only case for the heavy hole. The calculated scattering time for the exciton density of $n_{\mathrm{ex}}=$ $10^{15} \mathrm{~cm}^{-3}$ is $2.4 \times 10^{-9} \mathrm{~s}, 1.5 \times 10^{-9} \mathrm{~s}$, and $1.7 \times 10^{-10} \mathrm{~s}$ for the electron, light hole, and heavy hole, respectively, at $T=10 \mathrm{~K}$. The particular behavior of heavy-hole scattering times as measured using cyclotron resonance is probably a signature of significant exciton-carrier scattering. Finally, the dashed lines in Figs. 4(d)-4(f) correspond to the sum of (ni) and exciton scattering. By adding the contributions of $0.1 \% \mathrm{~N}_{\mathrm{ii}}$ ionized impurity scattering, as shown by the full lines, a reasonable consistency with the data (squares) is obtained.

Although we have not reached a perfect agreement, our first attempt to preliminarily determine the mobility appears to be successful at reproducing the general tendency. It is unique to diamond that both charge carriers and excitons coexist up to room temperature and above. As such a situation is realized only at cryogenic temperatures in prototypical semiconductors such as $\mathrm{Si}$ and $\mathrm{GaAs}$, further investigation of diamond at higher temperatures would be interesting. Studies toward the other extreme, i.e., higher-purity diamond at ultralow temperatures in the absence of neutral impurity scattering, would also be fascinating. When a higher sensitivity for detection is achieved and carrier-exciton scattering is suppressed, carrier mobility limited by isotope scattering might be observed for the first time in semiconductors.

\section{CONCLUSION}

We have quantified substitutional nitrogen and boron concentrations in several synthetic diamond crystals down to sub-ppb levels. The capture lifetimes of electrons and excitons and the carrier scattering times were discussed as a function of impurity concentrations. We extracted the cross section of electrons for boron impurity $\left(1 \times 10^{-14} \mathrm{~cm}^{2}\right)$, and that of excitons for nitrogen impurity $\left(3 \times 10^{-14} \mathrm{~cm}^{2}\right)$, which had been unknown so far for diamond. The scattering times of charge carriers with impurities, carbon isotopes, and excitons were quantitatively calculated, and the comparison with measured data indicated an almost complete neutralization of compensated impurities by photoexcited carriers.

The present study opens an avenue for the detailed understanding of dynamics of the coexistent system of charge carriers and excitons. The information obtained in the present study should be useful for predicting mobility-lifetime $(\mu \tau)$ products in intrinsic layers of diamond diodes and charge carrier collection efficiencies in diamond detectors.

\section{ACKNOWLEDGMENTS}

This work was partially supported by JSPS KAKENHI Grants No. 17H02910, No. 15K05129, and No. 26400317, 
and The Murata Science Foundation. N.N. thanks the French National Center for Scientific Research (CNRS) for financial support for an invited researcher position at GEMaC, Solange Temgoua (GEMaC, Versailles University
UVSQ) for cathodoluminescence measurements, Makoto Kuwata-Gonokami (The University of Tokyo) for providing the HPHT samples, and Yuzo Shinozuka (Wakayama University) for valuable discussion on alloy scattering.
[1] R. S. Balmer, J. R. Brandon, S. L. Clewes, H. K. Dhillon, J. M. Dodson, I. Friel, P. N. Inglis, T. D. Madgwick, M. L. Markham, T. P. Mollart, N. Perkins, G. A. Scarsbrook, D. J. Twitchen, A. J. Whitehead, J. J. Wilman, and S. M. Woollard, Chemical vapour deposition synthetic diamond: Materials, technology and applications, J. Phys.: Condens. Matter 21, 364221 (2009).

[2] T. Teraji, J. Isoya, K. Watanabe, S. Koizumi, and Y. Koide, Homoepitaxial diamond chemical vapor deposition for ultralight doping, Mater. Sci. Semicond. Process. 70, 197 (2017).

[3] N. Naka, J. Omachi, H. Sumiya, K. Tamasaku, T. Ishikawa, and M. Kuwata-Gonokami, Density-dependent exciton kinetics in synthetic diamond crystals, Phys. Rev. B 80, 035201 (2009).

[4] H. Sumiya and S. Satoh, High-pressure synthesis of high-purity diamond crystal, Diam. Relat. Mater. 5, 1359 (1996).

[5] H. Kawarada, H. Matsuyama, Y. Yokota, T. Sogi, A. Yamaguchi, and A. Hiraki, Excitonic recombination radiation in undoped and boron-doped chemical-vapor-deposited diamonds, Phys. Rev. B 47, 3633 (1993).

[6] J. Barjon, T. Tillocher, N. Habka, O. Brinza, J. Achard, R. Issaoui, F. Silva, C. Mer, and P. Bergonzo, Boron acceptor concentration in diamond from excitonic recombination intensities, Phys. Rev. B 83, 073201 (2011).

[7] J. Barjon, Luminescence spectroscopy of bound excitons in diamond, Phys. Status Solidi A 214, 1700402 (2017).

[8] R. Ulbricht, S. T. van der Post, J. P. Goss, P. R. Briddon, R. Jones, R. U. A. Khan, and M. Bonn, Single substitutional nitrogen defects revealed as electron acceptor states in diamond using ultrafast spectroscopy, Phys. Rev. B 84, 165202 (2011).

[9] J. Barjon, P. Valvin, C. Brimont, P. Lefebvre, O. Brinza, A. Tallaire, J. Achard, F. Jomard, and M. A. Pinault-Thaury, Picosecond dynamics of free and bound excitons in doped diamond, Phys. Rev. B 93, 115202 (2016).

[10] J. Barjon, E. Chikoidze, F. Jomard, Y. Dumont, M.-A. PinaultThaury, R. Issaoui, O. Brinza, J. Achard, and F. Silva, Homoepitaxial boron-doped diamond with very low compensation, Phys. Status Solidi A 209, 1750 (2012).

[11] K. Horiuchi, K. Nakamura, S. Yamashita, and M. KuwataGonokami, Photoluminescence characterization of high-purity synthesized diamond, Jpn. J. Appl. Phys. 36, L1505 (1997).

[12] J. A. van Wyk, E. C. Reynhardt, G. L. High, and I. Kiflawi, The dependences of ESR line widths and spin-spin relaxation times of single nitrogen defects on the concentration of nitrogen defects in diamond, J. Phys. D: Appl. Phys. 30, 1790 (1997).

[13] N. Naka, H. Morimoto, and I. Akimoto, Excitons and fundamental transport properties of diamond under photo-injection, Phys. Status Solidi A 213, 2551 (2016).

[14] I. Akimoto, N. Naka, and N. Tokuda, Time-resolved cyclotron resonance on dislocation-free HPHT diamond, Diam. Relat. Mater. 63, 38 (2016).

[15] N. Naka, K. Fukai, Y. Handa, and I. Akimoto, Direct measurement via cyclotron resonance of the carrier effective masses in pristine diamond, Phys. Rev. B 88, 035205 (2013).
[16] M. Fukai, H. Kawamura, K. Sekido, and I. Imai, Linebroadening of cyclotron resonance due to lattice and neutral impurity scattering in silicon and germanium, J. Phys. Soc. Jpn. 19, 30 (1964).

[17] J. Barjon, M.-A. Pinault, T. Kociniewski, F. Jomard, and J. Chevallier, Cathodoluminescence as a tool to determine the phosphorus concentration in diamond, Phys. Status Solidi A 204, 2965 (2007).

[18] H. Morimoto, Y. Hazama, K. Tanaka, and N. Naka, Exciton lifetime and diffusion length in high-purity chemicalvapor-deposition diamond, Diam. Relat. Mater. 63, 47 (2016).

[19] R. Sauer, N. Teofilov, and K. Thonke, Exciton condensation in diamond, Diam. Relat. Mater. 13, 691 (2004).

[20] T. Teraji, T. Yamamoto, K. Watanabe, Y. Koide, J. Isoya, S. Onoda, T. Ohshima, L. J. Rogers, F. Jelezko, P. Neumann, J. Wrachtrup, and S. Koizumi, Homoepitaxial diamond film growth: High purity, high crystalline quality, isotopic enrichment, and single color center formation, Phys. Status Solidi A 212, 2365 (2015).

[21] D. Yu Fedyanin and M. Agio, Ultrabright single-photon source on diamond with electrical pumping at room and high temperatures, New J. Phys. 18, 073012 (2016).

[22] P. J. Dean and D. C. Herbert, Excitons, edited by K. Cho (Springer, Berlin, 1979).

[23] I. Akimoto, Y. Handa, K. Fukai, and N. Naka, High carrier mobility in ultrapure diamond measured by time-resolved cyclotron resonance, Appl. Phys. Lett. 105, 032102 (2014).

[24] C. Erginsoy, Neutral impurity scattering in semiconductors, Phys. Rev. 79, 1013 (1950).

[25] E. Otsuka, K. Murase, and J. Iseki, Electron scattering by neutralized acceptors in germanium. I. Gallium and indium, J. Phys. Soc. Jpn. 21, 1104 (1966).

[26] S. Fahy and E. P. O'Reilly, Intrinsic limits on electron mobility in dilute nitride semiconductors, Appl. Phys. Lett. 83, 3731 (2003).

[27] T. Ruf, M. Cardona, H. Sternschulte, S. Wahl, K. Thonke, R. Sauer, P. Pavone, and T. R. Anthony, Cathodoluminescence investigation of isotope effects in diamond, Solid State Commun. 105, 311 (1998).

[28] D. L. Dexter and F. Seitz, Effects of dislocations on mobilities in semiconductors, Phys. Rev. 86, 964 (1952).

[29] B. K. Ridley, Quantum Processes in Semiconductors (Oxford University Press, Oxford, 1999).

[30] Given a $10^{4-5} \mathrm{~cm}^{-2}$ dislocation density, it would provide a negligible amount of charged centers compared with $N_{\mathrm{ii}}$.

[31] T. Ohyama, T. Sanada, and E. Otsuka, Time-resolved cyclotron resonance analysis of electron-exciton interaction in silicon, J. Phys. Soc. Jpn. 35, 822 (1973).

[32] S. G. Elkomoss and G. Munschy, Electron-exciton elastic scattering cross sections in the central field and the exchange approximations, J. Phys. Chem. Solids 38, 557 (1977). 